\title{
Hábitos posturais inadequados e dor em adolescentes
}

\author{
Inappropriate posture habits and pain in adolescents \\ Hábitos posturales inapropriados y dolor en adolescentes
}

Recebido: 10/07/2021 | Revisado: 17/07/2021 | Aceito: 21/07/2021 | Publicado: 28/07/2021

\author{
Daniela Aparecida de Faria \\ ORCID: https://orcid.org/0000-0001-8938-9371 \\ Universidade Federal de São João del-Rei, Brasil \\ E-mail: danielaffisio@hotmail.com \\ Paulo Henrique Nogueira da Fonseca \\ ORCID: https://orcid.org/0000-0002-2704-8923 \\ Universidade Federal de São João del-Rei, Brasil \\ E-mail: paulohenriquephn@gmail.com \\ Dayanne Gabriela de Melo Marques \\ ORCID: https://orcid.org/0000-0002-9394-1840 \\ Universidade Federal de São João del-Rei, Brasil \\ E-mail: dayannemelo10@hotmail.com \\ Kelly Aline Rodrigues Costa \\ ORCID: https://orcid.org/0000-0003-4289-1780 \\ Universidade Federal de São João del-Rei, Brasil \\ E-mail: kellyalinerodrigues@yahoo.com.br \\ Luísa Amanda Hermínia Martins \\ ORCID: https://orcid.org/0000-0002-8725-7372 \\ Universidade Federal de São João del-Rei, Brasil \\ E-mail: luisaamandabd_2@yahoo.com.br
}

\begin{abstract}
Resumo
Este estudo tem por objetivo, descrever os hábitos posturais inadequados e presença de dor em adolescentes escolares. Foi realizado um estudo transversal, exploratório, descritivo, com abordagem quantitativa. A amostra foi de 74 adolescentes, de 10 a 19 anos. A coleta de dados foi feita através da aplicação do Teste BackPEI (Back Pain and Body Posture Evaluation Instrument). A análise dos dados revelou a ocorrência de hábitos de posturas inadequadas nas atividades como ao ler na cama, ao sentar à mesa para escrever ou para conversar, ao utilizar o computador, ao pegar objetos no chão. A presença de dor esteve possivelmente associada às posturas inadequadas. Os resultados sugerem uma urgente necessidade do desenvolvimento de programas educativos e preventivos no âmbito escolar para a promoção e prevenção de dores corporais e hábitos posturais inadequados em adolescentes.
\end{abstract}

Palavras-chave: Dor; Saúde do adolescente; Educação em saúde; Saúde na escola.

\begin{abstract}
This study aimed to describe inappropriate postural habits and the presence of pain in adolescent students. A crosssectional, exploratory, descriptive study with a quantitative approach was carried out. The sample consisted of 74 adolescents, aged 10 to 19 years. Data collection was performed using the BackPEI Test (Back Pain and Body Posture Evaluation Instrument). Data analysis revealed the occurrence of habits of inappropriate posture in activities such as reading in bed, sitting at the table to write or talking, using the computer, picking up objects on the floor. The presence of pain was possibly associated with inadequate posture. The results suggest an urgent need to develop educational and preventive programs in the school environment for the promotion and prevention of bodily pain and describe inappropriate postural habits in adolescents.
\end{abstract}

Keywords: Pain; Teen health; Health education; Health at school.

\section{Resumen}

Este estudio tiene como objetivo describir los hábitos posturales inadecuados y la presencia de dolor en adolescentes escolares. Se realizó un estudio descriptivo, exploratorio, transversal con abordaje cuantitativo. La muestra estuvo formada por 74 adolescentes, de 10 a 19 años. La recolección de datos se realizo utilizando el BackPEI test (Instrumento de Evaluación de Dolor de Espalda y Postura Corporal). El análisis de datos reveló la ocurrencia de hábitos de postura inadecuada en actividades como leer en la cama, sentarse a la mesa a escribir o hablar, usar la computadora, recoger objetos del piso. La presencia de dolor posiblemente se asoció con posturas inapropiadas. Los resultados sugieren una urgente necesidad de desarrollar programas educativos y preventivos en el ámbito escolar para la promoción del hábitos posturales inadecuados y dolor corporal en adolescentes.

Palabras clave: Dolor; Salud los adolescentes; Educación para la salud; Salud en la escuela. 


\section{Introdução}

De acordo com a Organização Mundial da Saúde, a adolescência é delimitada cronologicamente como o período entre os 10 a 19 anos (Brasil, 2018). Nessa fase da adolescência ocorrem grandes transformações biopsicossocias, tornando o adolescente mais vulnerável (Beling et al., 2018), merecendo maior atenção nesse período.

Na adolescência, ossos, músculos e articulações encontram-se em processo de desenvolvimento, e nessa etapa, os hábitos posturais inadequados podem aumentar a chance do surgimento de dores que podem persistir na fase adulta (Cavalcante Filho et al., 2014; King Sara et al., 2011). Torna-se fundamental a detecção de padrões posturais inadequados para promover a saúde dos adolescentes e prevenir agravos relacionados ao desenvolvimento físico (Badaró, Nichele, \& Turra,2015).

A postura é a correlação do estado de equilíbrio entre ossos, músculos e articulações, com a capacidade para proteger as demais estruturas do corpo humano de traumatismos, seja na postura vertical (em pé), sentada, deitada, em movimento ou na forma estática (Cavalcante Filho et al., 2014). O adjetivo corporal qualifica o que é relativo ao corpo e a postura corporal qualifica a posição em que esse corpo se encontra, enquanto o hábito é um comportamento que, após ser repetido várias vezes, torna-se automático, isto é, realizado sem a deliberação prévia do indivíduo (Cristo, Günther, 2016). Considera-se as alterações posturais, principalmente as estáticas e na condição de hábito, como problema de saúde pública, de modo especial as que afetam a coluna vertebral, pois, podem predispor condições degenerativas na coluna vertebral na fase adulta (Vitta, Martinez, Piza, Simeão \& Ferreira, 2011; Silva Neto et al., 2021).

Segundo a Internacional Association for the Study of Pain - IASP, (Raja, et al., 2020), define-se dor como "a experiência sensitiva e emocional desagradável associada ou relacionada à lesão real ou potencial dos tecidos". Considera-se também que os fatores culturais e pessoais possam interferir na experiência e expressão da dor, onde cada pessoa aprende a referenciar o termo da dor através das suas experiências pessoais e culturais (IASP, 2010; Edwards, et al., 2016)

As atividades de vida diárias (AVDs) compreendem as várias atividades que circundam o indivíduo e fazem parte do seu modo de viver, tais como: mobilidade, comunicação, atividades laborativas, sono, dentre outras (Bezerra, Pagliuca \& Galvão, 2009; Farías-Antúnez, 2018). A fase da adolescência, as AVDs são vivenciadas em sua maior parte do tempo no ambiente escolar e em situação que exijam posturas estáticas, o que predispõe ao aparecimento de dor e de alterações posturais que podem repercutir em complicações na fase adulta (Vitta, Martinez, Piza, Simeão \& Ferreira, 2011). Neste contexto, torna-se importante identificar e descrever os hábitos posturais de adolescentes escolares, a fim de contribuir para o levantamento de dados no campo das políticas públicas de saúde voltadas para esse público (Sá, Carvalho \& Gomes, 2014; Eckhoff, Straume \& Kvernmo, 2017).

Desse modo, a pergunta de pesquisa foi embasada na hipótese de que adolescentes com a presença de hábitos posturais inadequados nas atividades de vida diárias tendem ao maior acometimento de dores musculoesqueléticas. Assim, o presente estudo objetivou identificar e descrever os hábitos posturais inadequados e presença de dor nas AVDs de adolescentes do ensino fundamental e médio de uma Escola Estadual do Município de Divinópolis/MG.

\section{Metodologia}

Trata-se de um estudo transversal, exploratório, descritivo, com abordagem quantitativa. O cenário do estudo foi uma escola estadual do Município de Divinópolis/MG, a amostra foi do tipo por conveniência, com a seleção de todos os adolescentes que se encontravam presentes na sala de aula e que preenchiam os critérios de inclusão.

A população foi composta por 300 adolescentes estudantes, dos quais 98 desejaram não participar do estudo, 54 faltaram no dia da aplicação; 58 não trouxeram o TCLE/TALE. Dessa maneira, a amostra foi de 74 adolescentes. O quantitativo estudado foi superior ao tamanho amostral mínimo calculado inicialmente por meio do programa Open Epi, versão 3.01, para um nível de confiança de $95 \%$, com erro amostral de $5 \%$ e poder estatístico de $80 \%$. 
Considerou-se como critérios de inclusão: (I) adolescentes regularmente matriculados na escola, (II) adolescentes presentes na sala de aula no dia e horário da coleta de dados, e; (III) adolescentes com 10 anos completos até 19 anos incompletos, após a assinatura dos Termos de Consentimento Livre e Esclarecido (TCLE) pelos responsáveis. Após orientação quanto as implicações do estudo, aqueles que consentiram foram instruídos a levar para casa o TCLE e a solicitar que os pais e/ou responsáveis o lessem e assinassem. Após o recolhimento do TCLE, os alunos eram instruídos a lerem e assinarem o Termo de Assentimento Livre e Esclarecido (TALE).

Para a coleta de dados foi utilizado o Teste BackPEI (Back Pain and Body Posture Evaluation Instrument), que tem sido empregado em vários estudos tanto a nível nacional quanto internacional (Noll et al., 2012). Trata-se de um instrumento validado na literatura científica e que é relevante para a avaliação de dor nas costas e de seus fatores de riscos (Noll et al., 2016). O teste configura-se como um questionário autoaplicável composto por 21 questões fechadas, com versões para o sexo feminino e masculino, específico para adolescentes escolares, que visa a avaliação da percepção corporal de escolares em relação à execução de suas AVDs. O questionário aborda os seguintes itens: (1) presença de dor nas costas nos últimos três meses (ocorrência, frequência e intensidade); (2) questões demográficas (idade e sexo); (3) questões socioeconômicas (escolaridade dos pais/responsáveis e tipo de escola); (4) questões comportamentais quanto às posturas nas realizações das AVDs (atividade física, ler/estudar na cama, horas/dia assistindo televisão e ao computador); (5) questões posturais (quanto ao modo de sentar para escrever e utilizar computador, quanto ao modo de transporte do material escolar, modo de dormir e modo de sentar para conversar) e; (6) questões hereditárias (ocorrência de dor nas costas nos pais/responsáveis) (Noll et al., 2016).

Após a aplicação dos questionários, as respostas foram transcritas para análise estatística utilizando o programa de estatística SPSS 21.0 (StatisticalPackage for the Social Sciences) para análises descritivas e de associação entre as variáveis. Para a análise descritiva foi realizado cálculo de média e desvio padrão para as variáveis quantitativas. Para calcular a significância estatística das associações, utilizou-se o teste qui-quadrado de Pearson $\left(\chi^{2}\right)$ com nível de significância estabelecido de 5\% ( $\leq \leq 0,05)$ para identificar possível associação entre as variáveis, sendo que as variáveis dependentes foram: postura adotada para escrever, postura sentada para utilizar o computador, postura para pegar um objeto no chão, posição de dormir, postura para conversar com os amigos, postura para carregar material, postura para levar uma mochila, escala de dor nas costas. As variáveis independentes foram: sexo, idade, IMC, escolaridade.

Em concordância à Resolução 466/12, foram aplicados o Termo de Consentimento Livre e Esclarecido (TCLE) e Termo de Assentimento (TA). Este estudo foi apreciado e aprovado pelo Comitê de Ética em Pesquisa da Universidade Federal de São João del-Rei, sob o número do parecer CAAE 60609316.1.0000.5545.

\section{Resultados e Discussão}

A amostra final foi de 74 adolescentes. O estudo evidenciou elevada incidência de dor física nos adolescentes e alterações posturais inadequadas nas AVDs.

Os principais resultados demonstraram, no que se refere às variáveis sexo, houve maior frequência do sexo feminino, 59,50\% ( $\mathrm{n}=44)$ para o masculino, 40,50\% $(\mathrm{n}=30)$; em relação à quantidade de horas que passa vendo TV, sobressaiu de 2 a 3 horas, $27,00 \%$ ( $\mathrm{n}=20$ ); já no item quantidade de horas no computador, houve maior ocorrência de 1 hora, 40,50\% (n=30), seguido de 2 a 3 horas, 24,3\% ( $\mathrm{n}=18)$. Quanto aos hábitos posturais inadequados nas AVDs, houve predominância nos hábitos: ler na cama, 32,40\% $(\mathrm{n}=24)$; postura de sentar à mesa da escola para escrever, 63,50\% $(\mathrm{n}=47)$, sentar na cadeira para conversar com os amigos, 66,20\% (n=49), sentar para utilizar o computador, 62,20\% ( $\mathrm{n}=46)$ e pegar objetos no chão, 66,20\% (n=49). Já quanto a presença de dor em algum local do corpo houve maior ocorrência de sim, 58,10\% (n=43) ao não, 25,70\% (n=19) com nível de significância de 0,059 usando ANOVA (Tabela 1). 


\section{Tabela 1.}

\section{ANOVA}

\section{Sexo}

\begin{tabular}{|l|r|r|r|r|r|}
\hline & \multicolumn{1}{|c|}{$\begin{array}{c}\text { Soma dos } \\
\text { Quadrados }\end{array}$} & df & \multicolumn{1}{c|}{$\begin{array}{c}\text { Muadrado } \\
\text { Médio }\end{array}$} & Z & Sig. \\
\hline Entre Grupos & 1,365 & 2 &, 682 & 2,941 &, 059 \\
Nos grupos & 16,473 & 71 &, 232 & & \\
Total & 17,838 & 73 & & & \\
\hline
\end{tabular}

Fonte: Autores.

Este estudo possibilitou conhecer a presença de dor em adolescentes escolares do ensino médio e fundamental de uma escola da rede estadual do município de Divinópolis/MG. Em uma amostra de 74 participantes, 58,1\% (n=43) relataram a presença de dor nas costas. Um estudo com o uso do mesmo instrumento do BackPei também apresentou a ocorrência significativa de dor em adolescentes escolares (Candotti, Roth, Noll, 2012). Torna-se importante dar visibilidade a esses dados, já que, na busca por alívio da dor, os adolescentes tendem a não fazem nada ou buscam automedicação, e, apenas uma pequena parcela do público, usa fármaco prescrito (Okamura, Madeira, Goldbaum \& Cesar, 2019).

Os resultados também demonstraram a possível associação entre dor com as posturas inadequadas nas AVDs relacionadas ao hábito de: ler na cama; a postura de sentar à mesa da escola para escrever; a sentar na cadeira para conversar com os amigos e para o uso do computador e pegar objetos no chão. Esses resultados corroboram os de outros estudos que correlacionam a prevalência de dor nas costas com as posturas inadequadas nas AVDs. No estudo de Noll et al. (2013), constatouse que dentre os 76 adolescentes escolares participantes da pesquisa, 65,8\% relataram a presença de dor nas costas e está esteve associada a posturas inadequadas nas AVDs. A manutenção dessas posturas inadequadas predispõe o aparecimento de lesões osteomusculares e o possível acometimento de dor. Resultados similares evidenciam que o aparecimento de dor nas costas na adolescência pode ser considerado um fator de risco para a dor nas costas na fase adulta, bem como outras possíveis alterações na coluna vertebral (Noll, Candotti, Vieira \& Fagundes, 2013; Meucci, 2018).

Similar aos resultados de outros estudos, no que se refere à variável gênero dos participantes, verificou-se incidência maior incidência no sexo feminino (Moreira, 2008; Yao, Mai, Luo, Ai \& Chen, 2011). A literatura tem demonstrado que os escolares do sexo feminino apresentam maior chance de ter dor nas costas e maior intensidade de dor em comparação com o sexo masculino (Yao et al., 2011). Sugere-se uma influência do fator social, relacionado ao padrão de normas comportamentais de gêneros ao relatar a dor, mostrando uma tendência do homem de subnotificar a dor e das mulheres de verbalizar e ter uma melhor percepção sobre ela. (Pool et al., 2007; Fowler et al., 2011; Alabas, Tashani, Johnson, 2013).

Investigações como as deste estudo são relevantes, visto que seus resultados possibilitam trabalhar a comunidade escolar no que refere à importância de prevenir dor nas costas (Yao et al., 2011) e por consequência, prevenir posturas inadequadas assumidas nessa etapa da vida, evitando impacto negativo na fase adulta. Alguns estudos com trabalhos de intervenção com adolescentes escolares, pais e educadores, obtiveram boa repercussão para uma psicoeducação e conscientização da manutenção da boa postura nas AVDs, com resultados apontando para a necessidade de programas educacionais que trabalhem hábitos posturais no âmbito escolar. (Biava \& Lima, 2009; Fernandes, Martins, Silva-Rocha \& Mendes, 2017). A baixa força de evidência para se estabelecer um consenso acerca dos valores de prevalência de desvios posturais de escolares brasileiros, reforça a necessidade de mais estudos para se chegar a dados e propostas mais concretas (Kasten, Rosa, Schmit, Noll \& Candotti, 2017).

Após a análise de todos os dados desta pesquisa, foram disponibilizados para a escola e aos adolescentes os resultados obtidos, juntamente com propostas para possíveis correções das inadequações nos hábitos posturais. Foram realizados com os 
adolescentes grupos de educação em saúde, que trouxeram como temática principal estratégias para radicar hábitos posturais adequados nas AVDs e medidas de prevenção de dor nas costas.

Como limitação do estudo, destacam-se: (I) a restrição do universo de uma escola pública para a investigação, dando margem para o questionamento da realidade em escolas privadas; (II) a importância de avaliar em estudos futuros o peso da mochila, ainda que este item não seja contemplado no BackPEI. Propõe-se que estudos qualitativos sejam acrescidos a este para avançar no entendimento do fenômeno quanto à presença de hábitos posturais inadequados e dor nas costas em adolescentes. Estudos desta natureza podem subsidiar o trabalho preventivo intersetorial no ambiente escolar.

\section{Considerações Finais}

Este trabalho contribuiu para apontar que há predominância nos hábitos inadequados nas AVDs e de dor em alguma parte do corpo entre os adolescentes do universo pesquisado. A presença inadequada de hábitos posturais pode colaborar para o surgimento de dor em adolescentes escolares. Não sendo tratadas precocemente, poderão agravar-se em possíveis complicações futuras, tanto na fase adulta quanto senil. $O$ entendimento é de que é eminente a necessidade de revisar as políticas públicas de saúde e educação, pensando no desenvolvimento de ações conjuntas destinadas à promoção e prevenção de dores corporais em adolescentes. Evidente que para isso, deve-se considerar a necessidade de profissionais que estejam preparados para identificar, avaliar os riscos e promover ações na conexão entre escola e setor de saúde.

\section{Referências}

Alabas, O. A., Tashani O. A., \& Johnson M. I. (2013). Effects of ethnicity and gender role expectations of pain on experimental pain: a cross-cultural study. Eur J Pain, 17(5), 776-86. 10.1002/j.1532-2149.2012.00229.

Badaró, A. F. V, Nichele, L. F. I. \& Turra, P. (2015). Investigação da postura corporal de escolares em estudos brasileiros. Fisioterapia e Pesquisa, 22(2), 197204. 10.590/1809-2950/13384622022015.

Beling, M. T. C., Ferreira, M. F. R., Araújo, A. M. M., Barros, A. F. S., Belling, G., \& Lamounier, J. A. (2012). Alterações na imagem corporal entre adolescentes do sexo feminino e fatores associados. Adolesc Saúde, 9(4), 11-18. 10.1590/1413-81232018238.12472016

Bezerra, C. P., Pagliuca, L. M. F. \& Galvão, M. T. G. (2009). Modelo de enfermagem baseado nas atividades de vida diária: adolescente diabética e deficiente visual. Esc Anna Nery, 13(4). 10.1590/S1414-81452009000400021.

Biava, J. M. S. \& Lima, D. F. (2009). Educação postural na escola: uma abordagem integradora do Programa de Desenvolvimento Educacional - PDE from http://www.diaadiaeducacao.pr.gov.br/portals/pde/arquivos/2371-8.pdf.

Brasil. Ministério da Saúde (2018). Proteger e cuidar da saúde de adolescentes na atenção básica. Ministério da Saúde, Secretaria de Atenção à Saúde, Departamento de Ações Programáticas e Estratégicas. Brasília: 2ed.

Candotti, C. T., Roth, E. \& Noll, M (2012). Evaluation of weight and mode of transport of student in school of education. Revista Paulista de Pediatria, São Paulo, 30(1), 100-6. 10.1590/S0103-05822012000100015.

Cavalcante Filho, D. E. A., Viana, C. N. L., Cabral, M. P. S. S., Cunha, F. V. M., Pacheco, F. S., Brito, A. K. S., Assis, R. C. \& Martins, M. C. C. (2014). Dor lombar em adolescentes: um rastreamento escolar. Journal of Human Growth and Development, 24(3), 347-352. http://pepsic.bvsalud.org/scielo.php?script=sci_arttext\&pid=S0104-12822014000300015\&lng=pt\&nrm=iso

Cristo, F. \& Günther, H. (2016). Como Medir o Hábito? Evidências de Validade de um Índice de Autorrelato. Psicologia: Teoria e Pesquisa, 32(2). $10.1590 / 0102-3772 \mathrm{e} 322224$.

Eckhoff, C., Straume, B., \& Kvernmo, S. (2017). Multisite musculoskeletal pain in adolescence and later mental health disorders: a population-based registry study of norwegian youth: the naahs cohort study. BMJ OPEN. 7 (2). 10.1136/BMJOPEN-2016-012035.

Edwards, R. R., Dworkin, R. H., Sullivan, M. D., Turk, D., Wasan, A. D. (2016). The role of psychosocial processes in the development and maintenance of chronic pain disorders. H H S Public Acess. 17 (9), 90-92. 10.1016/j.jpain.2016.01.001.

Farias-Antunez, Simone et al. Incapacidade funcional para atividades básicas e instrumentais da vida diária: um estudo de base populacional com idosos de Pelotas, Rio Grande do Sul, 2014. (2018). Epidemiol. Serv. Saúde [online]. 27 (2), e2017290. 10.5123/s1679-49742018000200005.

Fernandes, C. S., Martins, T., Silva-Rocha, N. \& Mendes, M. (2017). "Jogo da postura": programa de intervenção de enfermagem em crianças. Aquichan, 17(2), 195-203. 10.5294/aqui.2017.17.2.8.

Fowler, S. L., Rasinski, H. M., Geers AL, Helfer, S. G., France, C. R. (2011). Concept priming and pain: an experimental approach to understanding gender roles in sex-related pain differences. J Behav Med, 34(2), 139-47. 10.1007/s10865-010-9291-7. 
IASP. International Association for the Study of Pain (2010). Guia para tratamento da dor em contextos de poucos recursos. Translated from the original English edition, published in 2009 under the title Guide to Pain Management in Low-Resource Settings. 111 Queen Anne Ave N, Suite 501 Seattle, WA 98109-4955, USA. https://www.iasp-pain.org/files/Content/ContentFolders/Publications2/FreeBooks/GuidetoPainManagement_Portuguese.pdf.

Kasten, A. P., Rosa, B. N., Schmit, E. F. D., Noll, M. \& Candotti, C. T. (2017). Prevalência de desvios posturais na coluna em escolares: revisão sistemática com metanálisemeta-analysis. Journal of Human Growth and Development, 27(1), 99-108. 10.7322/jhgd.127684

King Sara, et al. (2011). The epidemiology of chronic pain in children and adolescents revisited: a systematic review. PAIN. 152 (12), 2729-2738. 10.1016/J.PAIN.2011.07.016.

Leonardo, M (2009). Antropologia da alimentação. Rev Antropos, 3. http://revista.antropos.com.br/downloads/dez2009/Artigo\%201\%20-\%20Anntro pologia\%20da\%20Alimenta\%E7\%E3o\%20-\%20Maria\%20Leonardo.pdf .

Meucci, R. D., Duarte, V. M., Olmedo, D. W. V., Linhares, A. Z., Sobrinho, E. L. P. C. \& Cesar, J. (2018). Dor lombar em adolescentes do semiárido: resultados de um censo populacional no município de Caracol (PI), Brasil. Ciência \& Saúde Coletiva, 23(3), 733-740. 10.1590/1413-81232018233.04312016.

Moreira, S. (2008). Características da postura corporal de escolares da rede municipal de ensino de Porto Alegre. Dissertação de Pós-Graduação em Ciência do Movimento como título de Mestre em Ciências do movimento humano. http://www.lume.ufrgs.br/handle/10183/14728.

Noll, M. Candotti, C. T., Tiggemann, C. L., Wolf, M. C. S., Vieira, A. (2013). Prevalência de hábitos posturais inadequados de escolares do ensino fundamental da cidade de Teutônia: Um estudo de base populacional. Rev Bras.Ciênc.Esporte, 35 (4), 983-1004, 10.1590/S0101-32892013000400012

Noll, M., Candotti, C. T., Rosa, B. N. \& Fagundes, L. J. (2016). Back pain prevalence and associated factors in children and adolescents: an epidemiological population study. Rev Saúde Pública, 50(31). 10.1016/ j.pmn.2017.10.005.

Noll, M., Candotti, C. T., Vieira, A, \& Fagundes, L. J. (2013). Back Pain and Body Posture Evaluation Instrument (BackPEI): Development, content validation and reproducibility. International Journal of Public Health, 58(4). 10.1007/s00038-012-0434-1.

Pool, G.J, Schwegler, A.F, Theodore, B.R, Fuchs, P.N. (2007). Role of gender norms and group identification on hypothetical and experimental pain tolerance. Pain. 129(1- 2), 122-9.

Okamura, M. N., Madeira, W. Goldbaum, M. \& Cesar L. G. (2019). Back pain in adolescents: prevalence and associated factors. BrJP, 2(4), 321-325. $10.5935 / 2595-0118.20190059$.

Raja, S. N., Carr, D. B., Cohen, M., Finnerup, N., Flor, H., Gibson, S., et al. (2020). The revised International association for the study of pain definition of pain: concepts, challenges, And compromises. PAIN. 23. 10.1097/J.PAIN.0000000000001939.

Sá, C. S., Carvalho, R. G. S. \& Gomes, L. E. (2014). Saúde escolar de crianças no ensino fundamental: avaliação da prevalência de dor nas costas. Arq Ciênc.Saúde, abr/jun, 21 (2), 77-82. Disponível em https://www.researchgate.net/publication/272164666

Silva N., A. C., Pereira, B. P. M., Sousa, J. S., França, G. L. M., Fernandes, R. J. \& Gouveia, N. M. (2021). Alterações posturais da coluna cervical e cervicalgia associadas ao perfil dos acadêmicos de medicina: uma revisão integrativa. Brazilian Journal of Development, 7(3). 10.34117/bjdv7n3-316.

Vitta, A., Martinez, M. G., Piza, N. T., Simeão, S. F. A. P. \& Ferreira, N.P. (2011). Prevalência e fatores associados à dor lombar em escolares. Cad Saúde Pública, 27. 10.5935/2595-0118.20180047

Yao, W., Mai, X., Luo, C., Ai, F., \& Chen, Q (2011). A cross-sectional survey of nonspecific low back pain among 2083 school children in China. Spine, 36 (22), 1885-90. 10.1097/BRS.0b013e3181faadea. PMID: 21270687. 